\title{
NEAR-INFRARED SPECTROSCOPY OF NGC 253: STARBURST AND SURROUNDINGS
}

\author{
D.LUTZ AND F.PRADA \\ Max-Planck-Institut für extraterrestrische Physik \\ Postfach 1603, 85740 Garching, Germany
}

Near-infrared longslit spectra of NGC 253 obtained with IRSPEC at the ESO NTT are presented. By analysis of the ${ }^{12} \mathrm{CO} 2.29 \mu \mathrm{m}$ bandhead we find that the stellar population in the central starburst region $(\mathrm{r} \sim 150 \mathrm{pc})$ rotates more slowly than the gas, but has a velocity dispersion of $128 \mathrm{~km} / \mathrm{s}$, about twice the value found for emission lines from the gas in this region. This implies an about five times higher dynamical mass than previously derived (Rieke et al. 1980), removing the need to invoke a lower mass cutoff in the starburst initial mass function. The peak of near-infrared emission is displaced from the dynamical center.

We discuss extinction values derived from line ratios of $[\mathrm{Fe} \mathrm{II],} \mathrm{H}$, and $\mathrm{H}_{2}$. While part of the differences can be explained by the wavelength dependency of derived extinction in case of mixed emitters and absorbers, there are also significant differences between the extinction for $\mathrm{H}$ and $\mathrm{H}_{2}$. This requires differences in local extinction and calls for caution in the interpretation of $\mathrm{H}$ vs. $\mathrm{H}_{2}$ line ratios in other starburst galaxies.

Along the major axis of the galaxy, the $\mathrm{H}$ and [FeII] emission are similarly distributed and trace the starburst region of radius about $150 \mathrm{pc}$. The $\mathrm{H}_{2}$ emission is more extended, reaching out to at least $300 \mathrm{pc}$. This halo of $\mathrm{H}_{2}$ emission which can be interpreted in terms shocks, which would have to be slow to avoid excitation of [FeII], or of a huge photon dominated region. The required properties of such a PDR - dense molecular clouds in a hot thin medium are consistent with properties inferred from Far-infrared observations (Carral et al. 1994). In none of the near-infrared lines do we find evidence for linesplits due to the NGC 253 superwind.

Carral,P., et al. 1994, Astrophys. J. 423,223

Rieke,G.H., et al. 1980, Astrophys. J. 238,40 